\title{
Evaluation of Business Solutions in Manufacturing Enterprises
}

\section{Yudistira Asnar*, Paolo Giorgini}

Dipartimento di Ingegneria e Scienza dell'Informazione,

University of Trento, Italy

\{yudis.asnar, paolo.giorgini\}@disi.unitn.it

* Corresponding author

\section{Paolo Ciancarini, Rocco Moretti}

Department of Information and Communication Technology,

University of Bologna, Italy

\{ciancarini, moretti\}@cs.unibo.it

\section{Maurizio Sebastianis}

Think3 Inc.

Maurizio.Sebastianis@think3.com

\section{Nicola Zannone}

Department of Computer Science, University of Toronto, Canada

zannone@cs.toronto.edu

\begin{abstract}
Evaluating business solutions before being deployed is essential for any organization. Risk is emerging as one of the most preeminent and accepted metrics for the evaluations of business solutions. In this paper, we present a comprehensive case study where the Tropos Goal-Risk framework is used to assess and treat risk on the basis of the likelihood and severity of failures within organizational settings. We present an analysis and an evaluation of business solutions within manufacturing enterprises.
\end{abstract}

Keywords: Risk, Dependability, Metrics, Organization

\section{Biography Notes:}

Yudistira Asnar is a PhD student in ICT School at University of Trento, Italy. $\mathrm{He}$ received his degree in Informatics Engineering from Institut Teknologi Bandung, Indonesia in 2002. His research interests lie in the area of requirement engineering, agent systems, and security-dependability risk management.

Paolo Giorgini is researcher at University of Trento. He received his Ph.D. degree from Computer Science Institute of University of Ancona (Italy) in 1998. He has worked on the development of requirements and design languages for agent-based systems, he is one of the founder of Tropos, an agent-oriented software engineering methodology. His publication list includes more than 140 refereed journal and conference proceedings papers and eight edited books. He is Co-editor in Chief of the International Journal of Agent-Oriented Software 
Engineering (IJAOSE).

Paolo Ciancarini is full professor in Computer Science at the University of Bologna (Italy). His research interests lie in the area of software engineering and formal methods.

Rocco Moretti received a Ph.D. in Computer Science at University of Bologna in 2005. He is currently a post-doctoral researcher in the area of Software Engineering affiliated to the Department of Computer Science of the University of Bologna (Italy). His research interests include software quality, software metrics, requirements engineering, system and software modeling languages.

Maurizio Sebastianis received a degree in Civil Engineering at University of Bologna in 1981. He is currently technical R\&D manager in the PDM/PLM area of Think3 Inc.

Nicola Zannone received a Ph.D. in Computer Science at University of Trento in 2007. He is currently a Postdoc in the Department of Computer Science at the University of Toronto. His research interests include computer security and formal verification and focus on the modeling and verification of security and trust aspects during the early requirements analysis in software engineering.

\section{Introduction}

Analysis and evaluation of business solutions is essential for every organization in order to develop and implement successful business strategies. This has spurred the definition of several metrics, such as in Cambridge Advanced Learner, where metrics are defined as a system of measurement. Maizlitsh \& Handler (2005) refine IT metrics into value delivery (e.g., cost reduction, increase in revenue, and reduction of risk) and process improvement. Value delivery metrics are intended to measure the quantity value, while metrics for process improvement (e.g., Capability Maturity Model) measure the continuity and quality of a process.

The last years have seen the emergence of risk as a metric for prioritizing events; see for instance DoD (1980). Risk, defined as the combination of the likelihood of an event and its consequences (ISO/IEC Guide 73, 2002), depicts the criticality of an event in disrupting the system. Typically, analysts assess the risk level of events toward the system and allocate the resources for safeguards according to their criticality. Risk is also used to perform cost-benefit analysis among available solutions, and choose the most cost-effective solution (i.e., the highest ratio between risk reduction and cost).

Many frameworks have been proposed for risk management (e.g., Bedford \& Cooke (2001), Butler \& Fischbeck (2001), den Braber et al. (2003), DoD (1980)). However, most of them overlook to analyze the organizational setting where the system operates. An organization can be conceived as a network of actors where an actor depends on others actors for fulfilling its goals. Risks are propagated between actors over dependencies and it may happen that different actor may associate a different level of risk to their duties. As a consequence, additional safeguards should be adopted to make acceptable the level of risk for every actor of the organization.

In this paper, we present a comprehensive case study of the application of the Tropos Goal-Risk (GR) framework (Asnar \& Giorgini, 2006, Asnar et al., 2008) for the analysis and evaluation of business solutions within manufacturing Small Medium Enterprises 
(SMEs). We present a modeling and an analysis process tailored to tool-supported risk assessment and treatment/safeguard selection within organizational settings. The framework extends the Tropos Goal Model (Giorgini et al., 2005) by adopting the idea of the three layers analysis introduced by Feather et al. (2005) in their Defect Detection and Prevention (DDP) framework. The three layers allow us to reason about uncertain events that may obstruct business goals and to evaluate the effectiveness of treatments in mitigating such events.

In this paper, we also evaluate the expressiveness of the modeling language and validate the formal framework against an industrial case study. We analyze the intramanufacturing integration model defined in the course of the TOCAI.IT project ${ }^{1}$ in collaboration with Think $3,{ }^{2}$ a consultancy company that provides business solutions (e.g., product development solutions, consulting services, and customer cares) for optimizing the product development processes of SMEs. In particular, starting from the core requirements of manufacturing SMEs elicited in the course of the project (Kiyavitskaya et al., 2007), we have identified the risks affecting intra-manufacturing SMEs, and analyzed and evaluated the treatments that are usually adopted in industry practices. In (Asnar et al., 2008), we have analyzed this application domain using a qualitative approach. In this paper, we employ a quantitative approach to assess the risk.

The paper is organized as follows. Next, we present the intra-manufacturing integration model of SMEs that is used as a running example throughout the paper $(\S 2)$. Afterward, we discuss related work ( $\$ 3)$, and continue with a brief introduction of the GR framework (\$4). We present the risk management process and illustrate step-by-step its application to the intra-manufacturing integration model (\$5). Finally, we discuss our experience in applying the GR framework to the case study (\$6) and conclude the paper (§7).

\section{Intra-Manufacturing SMEs}

An intra-enterprise integration model is characterized by different divisions within the same organization (or of several organizations with strong synergies) collaborating for reaching common objectives or for executing specific processes. In the manufacturing domain, the objective is the realization of a specific product. According to their business model, manufacturing SMEs can be distinguished into two main categories: productoriented companies and order-oriented companies. The first category is mainly involved in the production of a "relatively" fixed set of products that aim to fulfill the needs of a large market. Typically, SMEs, in this category, aim to manufacture a competitive product by trading-off its quality. In this setting, time-to-market is the main aspect to be considered. The second category concerns the production of a variable set of products depending on the specific requirements given by customers. The main objectives for these companies are that products shall meet the customer needs. We refer to a detailed presentation of the core requirements for manufacturing SMEs to Kiyavitskaya et al. (2007), whereas in the rest of the paper we mainly focus on the risk affecting such organizations.

Though both categories of SMEs have a similar organizational structure and are

${ }^{1}$ FIRB-TOCAI.IT RBNE05BFRK - http://www.dis.uniroma1.it/ tocai/

${ }^{2}$ The official web site is http://www.think3.com 
exposed to similar risks, they require different business solutions. This is because of the different level of risks (i.e., likelihood and severity) due to the different business model as well as to the different business objectives. For instance, the event engineering change request of a product occurs more often in the order-oriented setting comparing at the product-oriented one. Conversely, the risk of late for product delivery is more severe for the product-oriented companies because the goal of reduce time-to-market is valued more than in the order-oriented one.

In product-oriented companies, risk analysis has to deeply investigate aspects concerning how to "produce the product right", whereas in order-oriented companies the analysis considers the issues of producing the "right product" for a client. From a software engineering perspective, such concerns are similar to the concepts of verification and validation of a product, respectively. Verification considers the compliance of the final product with its requirements, whereas validation focuses on the acceptance of the final product by the client. In a product-oriented company, the acceptance process of the products by clients is not feasible due to the large set of products and clients to be considered. Similarly, it is meaningless for an order-oriented company to evaluate the compliance with the requirements of a product without considering its acceptance by a specific client.

\section{State of the Art}

Many approaches have been proposed for identifying products and systems enabling the coordination and cooperation in the intra-enterprise integration model for SMEs. For instance, Lindsey et al. (1990) have studied the relationship between the organizational strategy and the effect of IT. Their work points out that a responsive infrastructure enabling to adapt and accept changes is essential to the strategic effectiveness of IT. According to McFarlan (1984), IT might influence the competition by introducing barriers to the entrance of new competitors, changing the relationship between customers and suppliers, enlarging the basis of potential suppliers or strengthening the existent relationships, or even by creating new businesses. Pooley \& Wilcox (2000) have analyzed the application of IT for supporting the coordination of geographicallydistributed teams. The development of such distributed environments can support decision making processes by means of the exchange of ideas and discussion.

However, the solution (e.g., products or systems) identification process must be followed up by an evaluation step. In the area of IT metrics, there are several mature metrics that have been proposed to evaluate business solutions. Goodman (2004) defines a set of metrics for evaluating software solutions. The set spans from measuring the complexity of software (e.g., function point) to cost-estimation metrics. The author also presents guidelines to customize metrics with respect to the needs of specific organizations. Rico (2004) puts more emphasis in the economical aspects of alternatives proposing metrics, such as Return-of-Investment, Net Present value, and Break-even point. The motivation behind these metrics is that the solution is part of the investment and must generate values to the organization. Jaquith (2007) specifies some criteria for the definition of good security metrics (e.g., without subjective criteria, cheap to conduct, expressed in terms of a cardinal number or percentage, and not qualitative values with at least one unit of measure, and specific/relevant enough for decision-making). Based on such criteria, the authors propose security metrics that are intended to measure the 
security of a system and relate them to business values.

In the risk analysis domain, there are several models that attempt to assess uncertain events together with their likelihood and severity. For instance, Probabilistic Risk Analysis (PRA) (Bedford \& Cooke, 2001) is widely used to assess risks quantitatively, and FMECA (DoD, 1980) proposes qualitative values (i.e., frequent, reasonable probable, occasional, remote, and extremely unlikely). Events are prioritized using the notion of "loss expectancy" which is computed over the likelihood of events and their severity. This priority represents the criticality of an event. When resources are limited, analysts can decide to adopt countermeasures for mitigating events on the basis of their priority. Butler \& Fischbeck (2001) recognize that many factors (e.g., reliable, available, safe, etc.) can be critical for a system and each of them has its own risks. Based on this intuition, they propose Multi-Attribute Risk Assessment to improve the risk analysis process by considering multi-attributes. The framework simultaneously considers many factors like reliability, availability, safety and confidentiality by enabling analysts to find the right trade-off among these factors. The capability of choosing cost-effective countermeasures to deal with existing security threats using Multi-Attribute Risk Assessment is presented in (Butler, 2002). Feather (Feather, 2004) proposes Defect Detection and Prevention (DDP). DDP consists of a three layers model: Objectives, Risks, and Mitigation. Each objective has a weight that represents its importance; each risk has a likelihood of occurrence; whereas mitigation has a cost for its accomplishment (mainly resource consumption). Severity of a risk impacting an objective can be represented by an impact relation between the objective and the risk. A DDP model also specifies how to compute the level of objective achievement and the cost of mitigation. This calculation allows the evaluation of the impact of adopted countermeasures to better support the decision making process. DDP has also been integrated with other quantitative frameworks (e.g., FMECA (DoD, 1980), FTA (Stamatelatos et al., 2002)) to model and assess risks/failures. Though these proposals are adequate to estimate the level of risk of a system, they consider the risk of technical system and lack in modeling and analyzing social/organizational aspects of the system. Finally, the CORAS methodology (den Braber et al., 2003) combines UML and Unified Process to support a model-based risk assessment. In particular, it proposes an integrated system development and risk management process for security critical systems.

\section{Tropos Goal-Risk}

The Tropos Goal-Risk (GR) framework (Asnar et al., 2008) has been designed to assess the risk of uncertain events over organization strategies and to evaluate the effectiveness of treatments. It consists of a modeling framework, which is explained in this section, and a risk assessment and treatment process, which is detailed in the next section.

The modeling framework consists of three conceptual layers:

- Asset layer captures strategic interests of the stakeholders and what are the means to achieve them;

- Event layer represents uncertain events along their impacts to the asset layer;

- Treatment layer depicts treatments to be adopted in order to mitigate the risk of the event layer over the asset layer. 


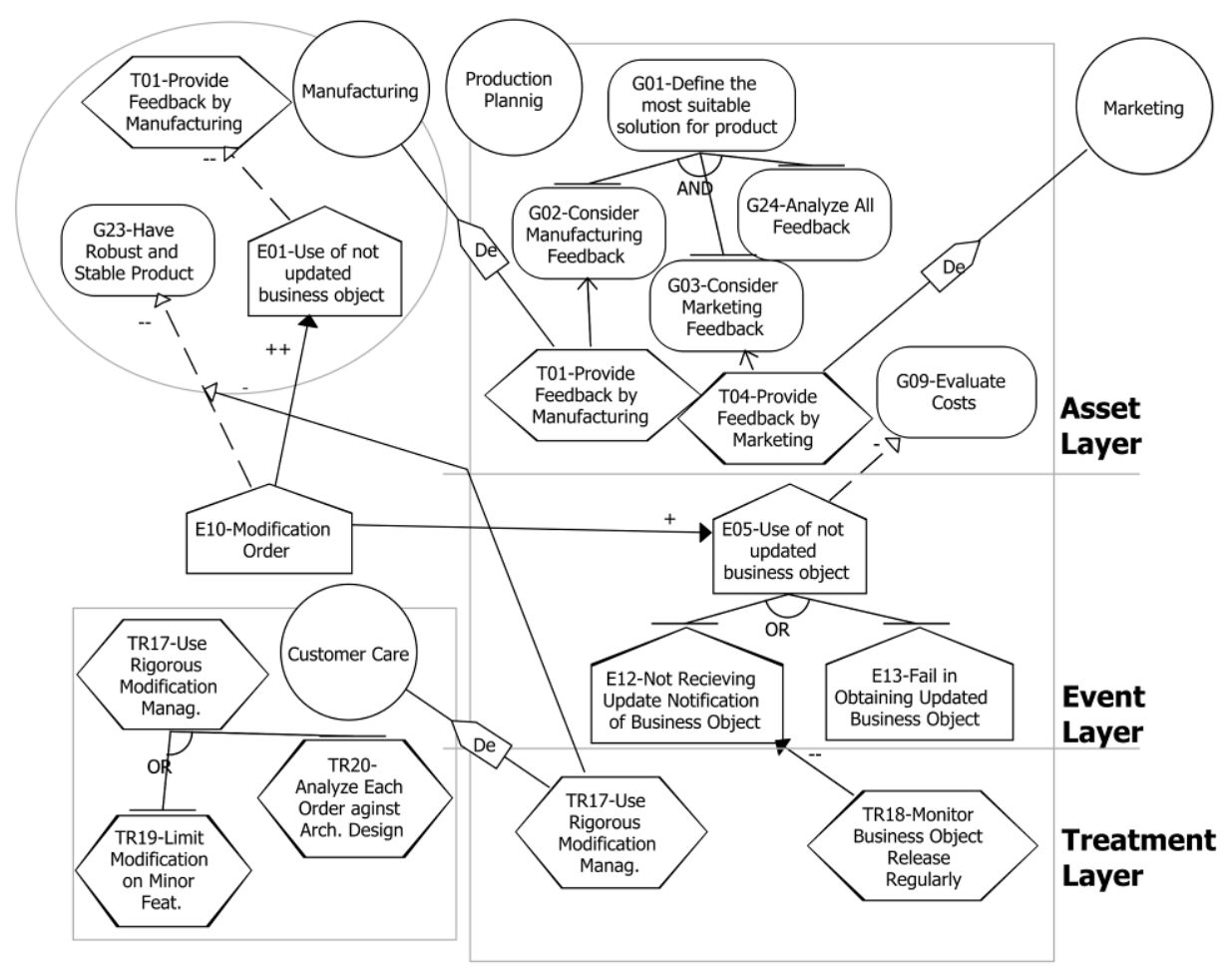

Figure 1: GR Model of Intra-Organization

A GR model (Figure 1) is a graph that consists of nodes and edges. Nodes are pairs where the first element is an actor and the second element is a goal, a task, or an event. Actors (depicted as circles) are active entities that have strategic goals and perform tasks to achieve them (e.g., Production Planning (PP), Manufacturing, Marketing, and Customer Care divisions in Figure 1). Goals (depicted as ovals) represent the objectives that actors intend to achieve (e.g., PP wants to define the most suitable solution for product $\left(\mathrm{G}_{01}\right)$. Tasks (depicted as hexagons) are courses of actions used to achieve goals (e.g., provide feedback by manufacturing $\left(\mathrm{T}_{01}\right)$ ) or to treat events (e.g., monitor business object release regularly $\left(\mathrm{T}_{18}\right)$ ). In the last case, tasks are called treatments to distinguish them to those providing meaning for the achievement of goals. Goals and tasks are characterized by attributes $S A T$ and $D E N$ which denote the evidence of satisfying and denying goals or tasks, respectively. Their value is defined in the range of [0, 1]. As shown in Figure 1, the PP division intends to achieve goal $G_{01}$; the evidence of $G_{01}$ in PP's viewpoint are denoted as $\operatorname{Sat}\left(\left\langle P P, \mathrm{G}_{01}\right\rangle\right)$ and $\operatorname{Den}\left(\left\langle P P, \mathrm{G}_{01}\right\rangle\right)$. Goals are also characterized by attribute utility (denoted by Utility $\left(\left\langle P P, \mathrm{G}_{01}\right\rangle\right)$ ). ) which represents the value generated by the fulfillment of goal $\mathrm{G}_{01}$ for $\mathrm{PP}$ and is defined in the range $[0,100]$. In this context, the loss (denoted by $\operatorname{Loss}\left(\left\langle P P, \mathrm{G}_{01}\right\rangle\right)$ ) is defined as the reduction of $\mathrm{G}_{01}$ utility for PP due to the occurrence of an event, and risk tolerance (denoted by $R T(\langle P P$, $\mathrm{G}_{01} /$ ) is the loss, concerning $\mathrm{G}_{01}$, that is acceptable by PP. Events (depicted as pentagons) 
are used to represent uncertain circumstances that can affect goals and tasks in the asset layer. An event is characterized by attribute likelihood, which represents the probability that an event occur, and its severity affecting the asset layer (captured by impact relations).

Edges consist of decomposition, contribution, means-end, impact, alleviation, and dependency relations. Two types of decomposition relation are possible: AND and OR decomposition. AND decomposition is used to refine goals, tasks, or events into more refined structures. For example, goal $\mathrm{G}_{01}$ is AND decomposed into subgoals $\mathrm{G}_{02}, \mathrm{G}_{03}$, and $\mathrm{G}_{24}$. This relation implies that all subgoals must be achieved to achieve $\mathrm{G}_{01}$. OR decomposition is used to model the alternatives to achieve a goal, to execute a task, or for the occurrence of an event. For instance, treatment $\mathrm{TR}_{17}$ has two alternatives of execution (OR-decomposition), either $\mathrm{TR}_{19}$ or $\mathrm{TR}_{20}$. Impact relations connect events to goals and tasks. These relations capture the severity of events over the asset layer and are distinguished into four types: + and ++ to model opportunities (i.e., events with positive impacts), and - and -- to model risks (i.e., events with negative impacts). For example, Event $\mathrm{E}_{05}$ obstructs goal $\mathrm{G}_{09}$. Means-end relations link goals to the tasks used to satisfy them. In Figure 1, $\mathrm{T}_{04}$ is the means to achieve $\mathrm{G}_{03}$. Contribution relations denote the side-effects of goals/tasks to the other goals/tasks/events. These relations are categorized into 4 types as impact relations. Additionally, these relations are also used to model the effect of treatments on the likelihood of events. For example, treatment $\mathrm{TR}_{18}$ is used to reduce the likelihood of event $\mathrm{E}_{12}$. Alleviation relations model the mitigation of the (negative) impact of events due to the adoption of some treatment. Alleviation relations are distinguished into: - and - - which represent the extent in mitigating the severity of events. In Figure 1, $\mathrm{TR}_{17}$ mitigates the severity of $\mathrm{E}_{10}$ obstructing $\mathrm{G}_{23}$. The formal semantics of these relations have been presented in (Asnar \& Giorgini, 2007).

The inter-dependencies among actors are modeled through dependency relations which denote the dependency an actor (the depender) to another actor (the dependee) for fulfilling a goal or executing a task (the dependum). In Figure 1, PP depends on Marketing for the execution of $\mathrm{TR}_{04}$. Intuitively, the evidence of $\mathrm{TR}_{04}$ in the PP's viewpoint is calculated from the evidence of Marketing about the same task. By means of the modeling language, one can assess the risks along the organization objectives and assets analysis. Next section presents the risk management process that includes the steps for eliciting GR models as well as for risk assessment.

\section{Tropos Risk Management Process}

In the previous section, we have presented a conceptual framework for risk identification, description, estimation, and treatment identification, within organizations. Here, we present step-by-step the risk management process (Figure 2) using themanufacturing SME example to illustrate the application of its phases. In this context, we will perform an of operationalization activity to refine an impricise concept to be measurable in specific observations.

The examples presented in this section are illustrator examples of the different steps of our approach in the context of the organizational domain for SMEs. The general scenario represented by these examples can be instantiated tailoring the steps included in the proposed approach for a specific organization. This can be done acquiring the organizational knowledge by interviewing the managers of the selected organization. 
However, such work is out of the scope of this paper.

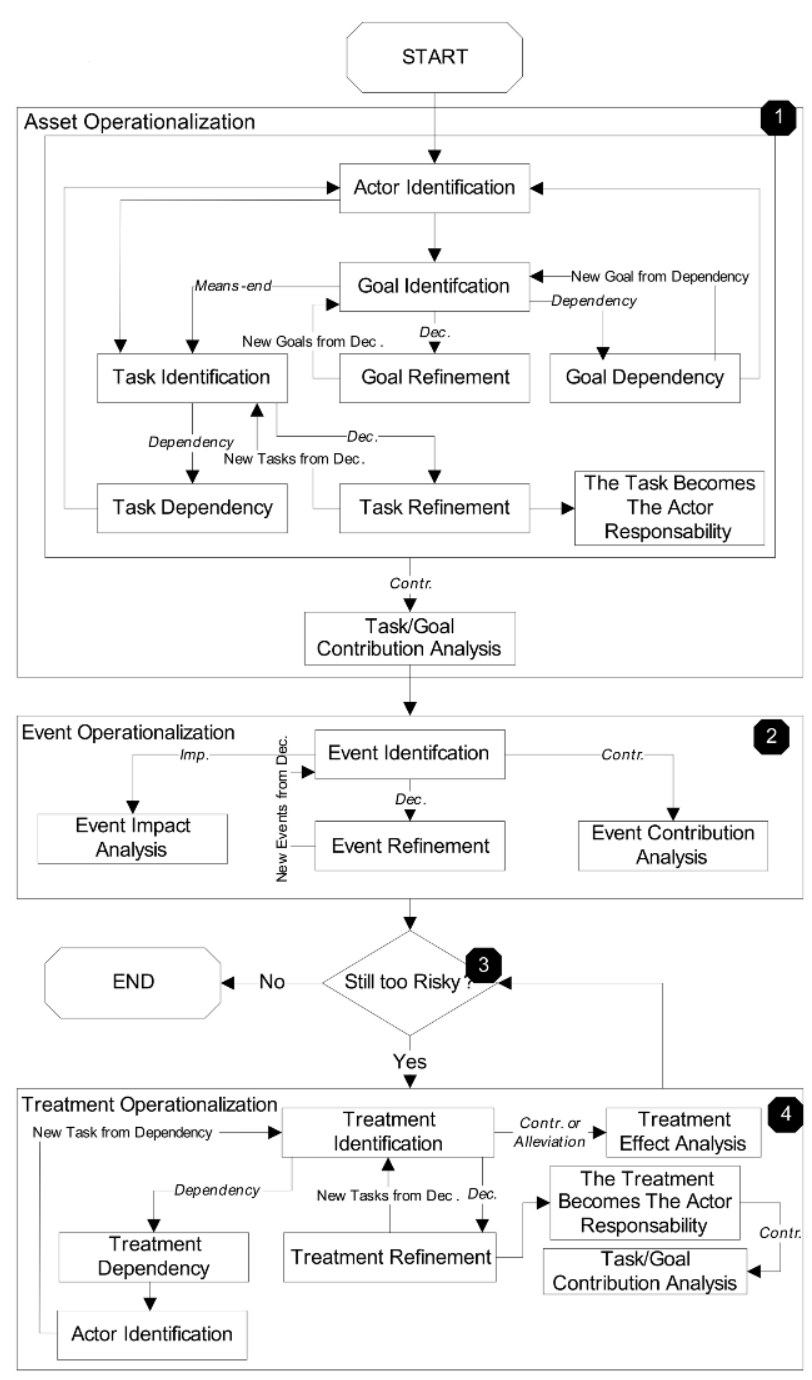

Figure 2: Tropos Risk Management Process

The process starts with (1) asset operationalization where assets of each actor are identified and analyzed in detail. Afterward, (2) event operationalization analyze the events that affect the identified assets; the likelihood and severity of every events is also assessed in this phase. Once business assets and events are analyzed, (3) risk assessment computes the risk level. If the risk level is beyond the specified risk tolerance, treatments can be introduced and analyzed during (4) treatments operationalization. Phases (3) and (4) are iterative, and the process stops when the risk level is acceptable by every actor. 


\subsection{Asset Operationalization}

Asset operationalization starts by identifying the actors relevant to the application domain. Actors are not only internal the organization, but also include external actors participating to the business activities of the organization such as clients and suppliers.

Example 1 - In the manufacturing SME domain, we have identified 9 main actors:

- $\quad$ The Chief Executive Officer (CEO) is responsible to ensure the global efficiency of the organization.

- $\quad$ The Customer Care division interacts with the customer.

- $\quad$ The Manufacturing division plans manufacturing activities and builds the products.

- $\quad$ The Marketing division defines the contents of the products and providing sales tools.

- $\quad$ The Production Planning division finds the most suitable solution for a product.

- $\quad$ The Purchase division defines and evaluates alternative purchase strategies.

- $\quad$ The Research and Development division develops new technologies and improves existing products.

- $\quad$ The Quality Assurance division tests products.

- $\quad$ The Sales division sells products to customers and supports them in the buying process.

The asset operationalization process may require the introduction of new actors to whom goals (or tasks) are delegated leading to a new iteration of actor identification.

For each identified actor, the analyst identifies its goals. Actors may not be able to fully achieve their goals by themselves. They can either appoint other actors to fulfill them entirely, or decompose them and assign part of them to other actors. Identified goals are thus used as input for goal refinement or goal dependency. 


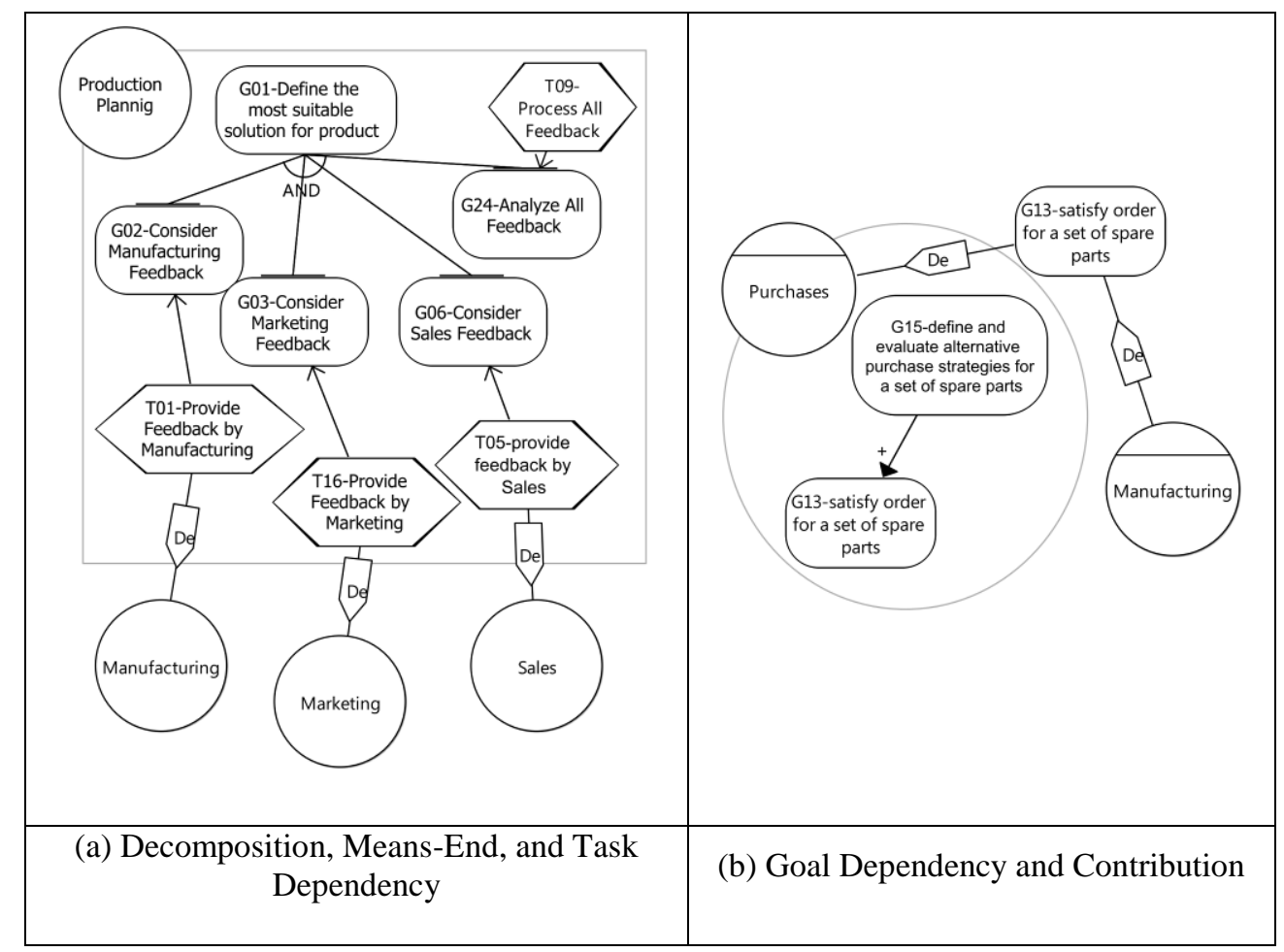

Figure 3: The Result of Asset Operationalization

Example 2 - As shown in Figure 3(a), the PP division is appointed to accomplish goal define the most suitable solution $\left(G_{01}\right)$. The division does not have the capabilities to fulfill $G_{01}$ by itself. Therefore, it decomposes into subgoals considering Manufacturing feedback $\left(G_{02}\right)$, considering Marketing feedback $\left(G_{03}\right)$, considering Sales feedback $\left(G_{06}\right)$, and analyze all feedback $\left(G_{24}\right)$.

Example 3 - The Manufacturing division is appointed to accomplish goal satisfy order for a set of spare parts $\left(\mathrm{G}_{13}\right)$ to guarantee the continuity of the manufacturing process. The division, however, is not able to fulfill the goal by itself and depends on the Purchases division for its fulfillment. This fragment of the requirements model is shown in Figure 3(b).

The tasks providing means for achieving goals are also identified in this phase. They are analyzed in a manner similar to that for goals.

Example 4 - As shown in Figure 3(a), the PP division achieves $G_{02}$ by means of task provide feedback by Manufacturing $\left(\mathrm{T}_{01}\right)$. For this task, the division depends on the Manufacturing division to execute it.

Dependency relations result in a new goal/task in the viewpoint of the delegatee and, consequently, require further analysis (i.e., refinement, means-end). However, there is a difference between goal and task dependency. Depending on an actor for achieving a goal implies that the dependee can decide the means (i.e., tasks) to fulfill the dependum. 
Conversely, depending on an actor for executing a task means that the depender has determined the exact procedure to be followed by the dependee for executing the task. In other words, the depender specifies "how-to" perform the assigned duty.

The first step of the asset operationalization process ends when the goals of every actor have been assigned to the capable actors. Next step consists in analyzing the sideeffects of a goal/task over other goals/tasks using contribution analysis.

Example 5 - As shown in Figure 3(b), the satisfaction of define and evaluate alternative purchase strategies for a set of spare parts $\left(G_{15}\right)$ positively contributes to the achievement of $\mathrm{G}_{13}$.

Table 1: Final Results of the Asset Operationalization

\begin{tabular}{|c|c|c|c|c|}
\hline & & Utility-Order & Utility-Product & Input-SAT \\
\hline \multicolumn{5}{|l|}{ CEO } \\
\hline G17 & reduce time-to-market & 40 & 70 & 0.9 \\
\hline & global efficiency* & 60 & 80 & \\
\hline \multicolumn{5}{|c|}{ Customer Care } \\
\hline T02 & provide feedback by Customer care & & & 0.8 \\
\hline \multicolumn{5}{|c|}{ Manufacturing } \\
\hline G13 & satisfy order for a set of spare parts* & 40 & 20 & \\
\hline G14 & test product* & 20 & 40 & \\
\hline & provide feedback by Manufacturing & & & 0.8 \\
\hline \multicolumn{5}{|c|}{ Marketing } \\
\hline G18 & global efficiency & 70 & 50 & 0.8 \\
\hline G19 & evaluate the impact on the market for the product & 70 & 80 & 0.8 \\
\hline G20 & define contents of the product & 70 & 80 & \\
\hline G21 & define functional contents of the product & & & 0.9 \\
\hline G22 & define non functional contents of the product & & & 0.85 \\
\hline T16 & provide feedback by Marketing & & & 0.8 \\
\hline \multicolumn{5}{|c|}{ Production Planning } \\
\hline G01 & define the most suitable solution for product & 80 & 60 & \\
\hline G02 & consider Manufacturing feedback & & & \\
\hline G03 & consider Customer Care feedback & & & \\
\hline G04 & consider R\&D feedback & & & \\
\hline G05 & consider Marketing feedback & & & \\
\hline G06 & consider Sales feedback & & & \\
\hline G07 & correct solution for product & 90 & 80 & \\
\hline G08 & evaluate costs & & & 0.85 \\
\hline G10 & evaluate available resources & & & \\
\hline G11 & reduce costs & 70 & 80 & 0.8 \\
\hline G12 & maximize use of solutions & 60 & 80 & 0.85 \\
\hline G24 & Analyze All Feedback & & & 0.8 \\
\hline T01 & provide feedback by manufacturing* & & & \\
\hline T02 & provide feedback by customer care* & & & \\
\hline т03 & provide feedback by $R \& D^{*}$ & & & \\
\hline T05 & provide feedback by Sales* & & & \\
\hline T06 & correction of the solution for product & & & \\
\hline T07 & other(s) & & & 0.75 \\
\hline T08 & define production plan & & & 0.85 \\
\hline T16 & provide feedback by Marketing* & & & \\
\hline \multicolumn{5}{|c|}{ Purchases } \\
\hline & satisfy order for a set of spare parts & & & 0.75 \\
\hline G15 & define and evaluate alternative purchase strategies for a set of spare parts & 40 & 40 & 0.6 \\
\hline \multicolumn{5}{|c|}{ Quality Assurance } \\
\hline G14 & test product & & & 0.8 \\
\hline \multicolumn{5}{|c|}{ Research and Dev. } \\
\hline & provide feedback by $R \& D$ & & & 0.8 \\
\hline \multicolumn{5}{|c|}{ Sales } \\
\hline G16 & increase sales & 90 & 90 & 0.9 \\
\hline T05 & provide feedback by Sales & & & 0.8 \\
\hline
\end{tabular}


Table 1 presents the identified actors together with their goals and the tasks employed to fulfill them. The symbol "*” indicates that the asset is delegated to another actor. Business (top) goals (indicated in bold in the table) are described along their utility with respect to the perspective of the corresponding actor. The utility represent how much a goal is critical for an actor. As suggested by Think3, asset utility can change depending on the business strategy adopted by the organization.

Example 6 - As shown in Table 1, goal reduce time-to-market $\left(\mathrm{G}_{17}\right)$ has a higher value for product-oriented companies, whereas goal define the most suitable solution for product $\left(\mathrm{G}_{01}\right)$ has a higher value for order-oriented companies. Accordingly, $\mathrm{G}_{17}$ is more critical in product-oriented companies than in order-oriented companies and viceversa for $\mathrm{G}_{01}$.

Goals and tasks can also be described in terms of their $S A T$ and $D E N$ evidence. In this case, we have specified $S A T$ evidence (column Input-SAT in Table 1) based on the Think3's past experiences. This evidence is used, together with relations among goals and tasks, and dependency relations, by the risk assessment process (Section 5.3) to assess the final evidence of the business goals.

\subsection{Event Operationalization}

Event operationalization is intended to analyze events and their impact on the asset layer. First, the process identifies the events relevant for the application domain and models them in the event layer. In this work, we have followed the guidelines defined in (Landwehr et al., 1994, Carr et al., 1993, Stamatelatos et al., 2002, Lee et al., 2006, Holton, 2004) for the identification of the events whose occurrence is critical to organizations, beside exploiting the knowledge of Think3 about the application domain.

Example 7 - Event of use of not updated business object $\left(\mathrm{E}_{01}, \mathrm{E}_{05}\right)$ and modification order $\left(\mathrm{E}_{10}\right)$ are critical events for manufacturing SMEs (Figure 4).

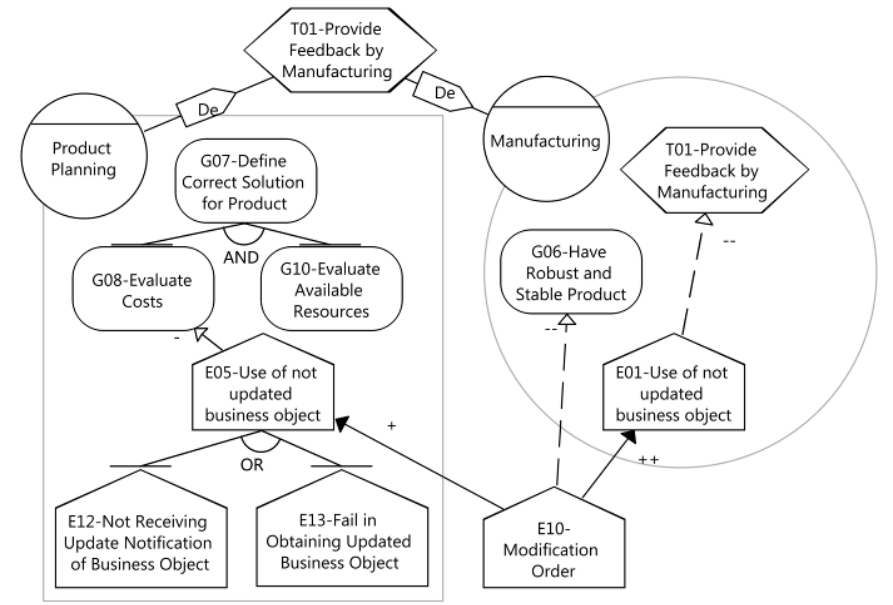


Figure 4: The Result of Event Operationalization

The reader may notice that events $\mathrm{E}_{01}$ and $\mathrm{E}_{05}$ have the same name. We noticed that the likelihood of events are different depending on in which actors it happens. Thus, the framework treats them as distinct events to avoid misunderstanding in the value of evidences. Conversely, all actors within the organization expose to $E_{10}$ with the same likelihood. To distinguish events on the basis of their behavior, we call local events for the events whose likelihood is related to an actor, and global events the events whose likelihood is independent from a single actor. In graphical models, local events are modeled inside the rational of actors; whereas global events are modeled outside the rational of actors (see Figure 4).

Events can be refined into finer events using decomposition relations (AND/OR). The refinement process stops when all leaf-events are tangible (i.e., can be easily assessed or their decomposition is negligible). This is similar to the proposal by Stamatelatos et al. (2002) who refine failure-modes/events until reaching basic events for which the data about their reliability is available and further refinement does not make meaningful difference.

Example 8 - Event $E_{05}$ is refined into events not receiving update notification of business object $\left(E_{12}\right)$ and fail in obtaining updated business object $\left(E_{13}\right)$. Conversely, $\mathrm{E}_{01}$ is not refined because Manufacturing does not distinguish $\mathrm{E}_{01}$ in finer events.

Events are also analyzed through contribution analysis to capture the interdependence among them. Table 2 lists the events relevant to the application domain and summarizes the relations between them.

Table 2: Relation among Events

\begin{tabular}{|c|c|c|c|c|c|c|c|c|c|c|c|}
\hline & & E01 & E02 & E03 & E04 & E05 & E07 & E08 & E09 & E10 & E11 \\
\hline \multicolumn{12}{|c|}{ Global Event } \\
\hline & Engineering Change Request & \multirow{6}{*}{+} & \multirow{6}{*}{+} & \multirow{6}{*}{+} & \multirow{6}{*}{+} & \multirow{6}{*}{+} & & \multirow{6}{*}{\multicolumn{2}{|c|}{+}} & \multirow[t]{6}{*}{++} & \multirow{6}{*}{++} \\
\hline E07 & production error & & & & & & & & & & \\
\hline E08 & not respected deadline for the delivery of a product & & & & & & & & & & \\
\hline E09 & spare part not found in the warehouse & & & & & & & & & & \\
\hline E10 & modification order & & & & & & & & & & \\
\hline E11 & test failure and/or quality check failure & & & & & & & & & & \\
\hline \multicolumn{12}{|c|}{ Manufacturing } \\
\hline \multicolumn{12}{|c|}{ E01 use of not updated business object } \\
\hline \multicolumn{12}{|c|}{ Marketing } \\
\hline \multicolumn{12}{|c|}{ E04 use of not updated business object } \\
\hline \multicolumn{12}{|c|}{ Production Planning } \\
\hline E05 & use of not updated business object & & & & & & + & & & & \\
\hline E12 & not receiving update notification of business object & & & & & OR & & & & & \\
\hline E13 & fail in obtaining updated business object & & & & & OR & & & & & \\
\hline \multicolumn{12}{|c|}{ Quality Assurance } \\
\hline E02 & use of not updated business object & & & & & & & & & & + \\
\hline \multicolumn{12}{|c|}{ Sales } \\
\hline E03 & use of not updated business object & & & & & & + & & & & \\
\hline
\end{tabular}

Table 3: Events Likelihood and Impacts toward the Asset Layer 


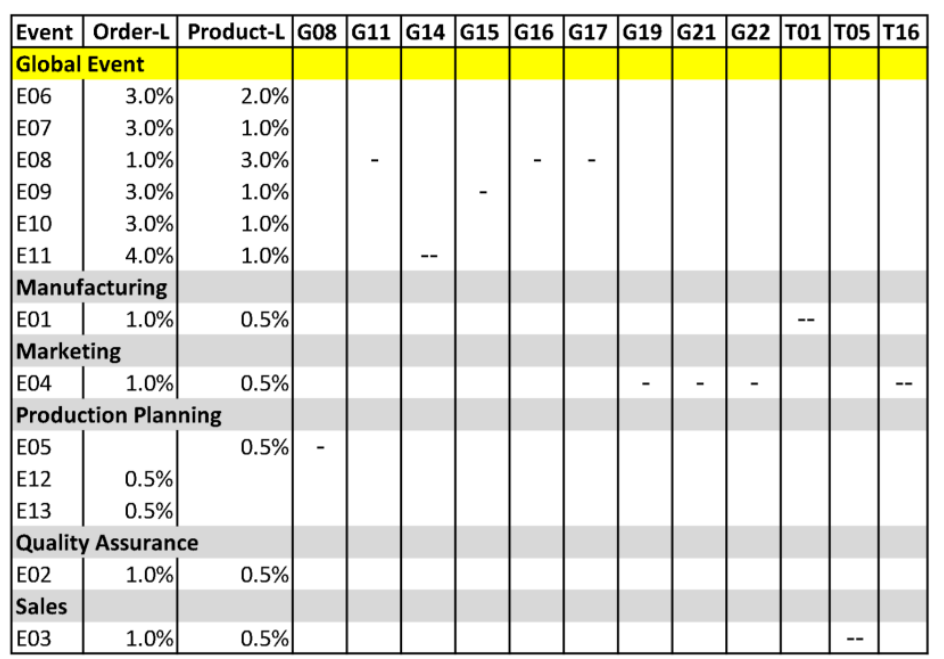

The impact of events over the asset layer is described and their likelihood estimated. Table 3 shows the likelihood and severity of events over the asset layer. These values have been defined according to the Think3's knowledge of the application domain. As done for asset utility, we have distinguished between order-oriented and product-oriented companies as the likelihood is characterized by the business strategy. Notice that events can have multi-impacts toward the asset layer. This allows analysts to perform trade-off analysis, especially over events that act as a risk (i.e., negative impact) for some goals and, at the same time, as an opportunity (i.e., positive impact) for other goals.

\subsection{Risk Assessment}

The risk assessment phase is intended to evaluate whether the risk level is acceptable for every actor. In other words, this phase verifies if the (expected) loss introduced because of risks is within the risk tolerance ${ }^{1}$ specified by every actor. To assist analysts in performing risk assessment, we have developed a quantitative reasoner ${ }^{2}$ that computes the risk for every actor within an organization and evaluate if it is within the specified risk tolerance. The reasoner implements the algorithm proposed in (Asnar et al., 2008).

In a nutshell, the reasoner computes the final $S A T$ and $D E N$ evidence of business goals for a given set of inputs. In particular, it takes in input a GR model together with the evidence, initially specified by the analyst, characterizing the asset layer (column Input- SAT Table 1) and the utility of business goals (columns Utility-Order and UtilityProduct in Table 1). The reasoner also requires the likelihood of events (columns Order-L and Product-L in Table 3) and the risk tolerance of every top goals depending on the actor viewpoint (column $R T$ in Table 4). The loss is then computed as the reduction of utility value on the basis of the evidence returned by the reasoner, as follows:

$$
\operatorname{Loss}(\langle A, G\rangle)=\operatorname{Utility}(\langle A, G\rangle) \times(1-[\operatorname{Sat}(\langle A, G\rangle)-\operatorname{Den}(\langle A, G\rangle)])^{3}
$$

\footnotetext{
${ }^{1}$ The risk tolerance criteria can be defined at the organization level or for each actor in the organization.

${ }^{2}$ http://sesa.disi.unitn.it/sistar tool

${ }^{3}[x]$ is a function where $[x]=x$ if $x>0$; otherwise $[x]=0$
} 
where $\operatorname{Utility}(\langle A, G\rangle)$ is the value specified by the analyst for business goal $G$ with respect to actor $A$, and $\operatorname{Sat}(\langle A, G\rangle)$ and $\operatorname{Den}(\langle A, G\rangle)$ are the evidence returned by the reasoner.

Table 4 shows the result of the assessment process. We use a "check" to indicate that the loss of a business goal is acceptable for the actor (i.e., $\operatorname{Loss}(\langle A, G\rangle) \leq R T(\langle A, G\rangle))$ and a "cross" to indicate that the loss of a business goal is not acceptable for the actor (i.e., $\operatorname{Loss}(\langle A, G\rangle>R T(\langle A, G\rangle))$.

Table 4: The Result of Risk Calculation

\begin{tabular}{|c|c|c|c|c|c|c|}
\hline & \multicolumn{3}{|c|}{ Order-Oriented } & \multicolumn{3}{|c|}{ Product-Oriented } \\
\hline & Utility & RT & Loss & Utility & RT & Loss \\
\hline \multicolumn{7}{|l|}{ CEO } \\
\hline G17 & 40 & 5 & $5.60 \times$ & 70 & 7 & $9.10 \times$ \\
\hline G18 & 60 & 15 & $14.40 \leadsto$ & 80 & 16 & $18.40 \times$ \\
\hline \multicolumn{7}{|c|}{ Manufacturing } \\
\hline G13 & 40 & 13 & $11.20 \leadsto$ & 20 & 5.3 & $5.40 \times$ \\
\hline G14 & 20 & 4 & $4.80 \times$ & 40 & 8.5 & $8.80 \times$ \\
\hline \multicolumn{7}{|c|}{ Marketing } \\
\hline G18 & 70 & 15 & $16.10 \times$ & 50 & 10.4 & $11.00 \times$ \\
\hline G19 & 70 & 15 & $16.10 \times$ & 80 & 16.5 & $17.60 \times$ \\
\hline G20 & 70 & 14 & $12.60 \leadsto$ & 80 & 13 & $13.60 \mathrm{X}$ \\
\hline \multicolumn{7}{|c|}{ Production Planning } \\
\hline G01 & 80 & 18 & $18.40 \times$ & 60 & 15 & $13.20 \leadsto$ \\
\hline G07 & 90 & 25 & $25.20 \times$ & 80 & 21 & $21.60 \times$ \\
\hline G11 & 70 & 14 & $16.80 \times$ & 80 & 16 & $18.40 \times$ \\
\hline G12 & 60 & 9 & $8.40 \leadsto$ & 80 & 12 & $11.20 \leadsto$ \\
\hline \multicolumn{7}{|c|}{ Purchases } \\
\hline G15 & 40 & 16 & $17.20 \times$ & 40 & 20 & $16.80 \leadsto$ \\
\hline \multicolumn{7}{|l|}{ Sales } \\
\hline G16 & 90 & 20 & $21.60 \times$ & 90 & 20 & $20.70 \times$ \\
\hline
\end{tabular}

In this work, we have analyzed the risks affecting two types of manufacturing SMEs: order-oriented companies and product-oriented companies. According to Think3's experience, those types of companies can define a different utility for the same goal and, consequently, suffer risks (due to the same event) differently.

Example 9 - As shown in Table 4, the Manufacturing division in order-oriented companies suffers loss affecting goal satisfy order for a set of spare parts $\left(\mathrm{G}_{13}\right)$ (i.e., $\operatorname{Loss}\left(\left\langle\right.\right.$ Manufacturing, $\left.\left.\left.G_{13}\right\rangle\right)=11.2\right)$ more than two times what the same division in product-oriented companies suffers (e.g., $\operatorname{Loss}\left(\left\langle\right.\right.$ Manufacturing, $\left.\left.\left.G_{13}\right\rangle\right)=5.4\right)$. Nonetheless, $G_{13}$ is more risky for product-oriented companies than for order-oriented companies. The product-oriented companies are more sensitive to the failure of $\mathrm{G}_{13}$. In other words, order-oriented companies define the risk tolerance over $\mathrm{G}_{13}$ higher than product-oriented companies do. In particular, risk tolerance $R T$ ( $\left.\left(M a n u f a c t u r i n g, G_{13}\right\rangle\right)$ is equal to 13 for order-oriented companies and to 5.3 for product-oriented companies.

For each goal whose expected loss is higher than the risk tolerance specified by the actor, analysts must mitigate the risk by introducing treatments. Once treatments are operationalized (Section 5.4), the risk assessment step is re-executed to verify whether residual risk (i.e., the loss after the adoption of treatments) is within the specified risk tolerance.

\subsection{Treatment Operationalization}

The treatment operationalization phase is intended to analyze the treatments to be 
adopted by an organization in order to mitigate assessed risks. This step shares the same conceptual and methodological approach used to analyze tasks during the asset operationalization. First, treatments are identified along the actors responsible to execute them; then treatments can be either delegated to other actors or refined. During this phase, new actors can be identified. Afterward, analysts assess the effect of treatments in mitigating the risk (either reducing the likelihood of events or reducing their severity).

Example 10 - As shown in Table 4, the loss affecting goal evaluate the impact on the market for the product $\left(\mathrm{G}_{19}\right)$ exceeds the risk tolerance defined by the Marketing division. The analysis performed during event operationalization has revealed that this situation is caused by event use of not updated business object $\left(\mathrm{E}_{04}\right)$. Think 3 has identified two treatments to reduce the likelihood of $\mathrm{E}_{04}$ : notify changes $\left(\mathrm{TR}_{14}\right)$ and find the last version of business object $\left(\mathrm{TR}_{15}\right)$. The execution of both treatments is assigned to the Marketing division. However, $\mathrm{TR}_{15}$ mitigates more effectively ( - contribution) than $\mathrm{TR}_{14}$ (- contribution). In addition to those treatment, Think3 has identified treatment know and apply concepts of effectiveness and versioning $\left(\mathrm{TR}_{10}\right)$ to reduce the likelihood of $\mathrm{E}_{04}$, whose execution is expected to be performed by the Warehouse division.

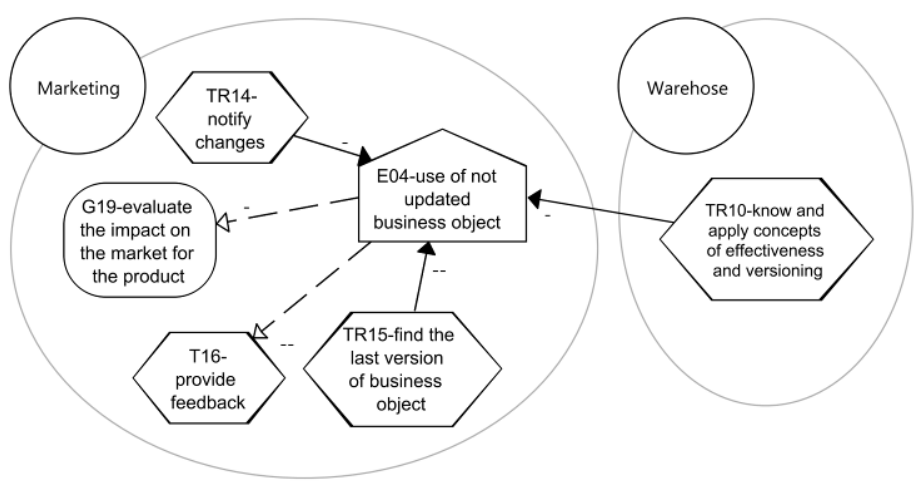

Figure 5: The Result of Treatment Operationalization

The reader may notice that Warehouse does not appear in the list of relevant actors (Example 1 - ). The reason is that Think3 has not reputed such a division relevant to the scenario initially. The analysis of risks and their treatments, on the contrary, has revealed that Warehouse can support the other divisions in reducing the risk of using not updated business object. The reduction of the severity of events is captured through alleviation relations as shown in the following example.

Example 11 - Figure 1 shows that the PP division employs treatment use rigorous modification management $\left(\mathrm{TR}_{17}\right)$ to alleviate the severity of global event modification order $\left(\mathrm{E}_{10}\right)$ over have robust and stable product $\left(\mathrm{G}_{23}\right)$.

During treatment operationalization, the side-effect of treatments over the asset layer is also analyzed. This step allows analysts to ensure that treatments do not introduce any unacceptable negative effect to goals or tasks. Finally, each treatment is associated with the cost of its adoption. The complete list of treatments and their cost is presented in 
Table 5. Notice that we have not distinguished the cost of treatments with respect to order-oriented and product-oriented companies. According to Think3, the cost is independent from the business strategy adopted by a company.

Table 5: List of Available Treatment Along with Two Strategies (A1 \& A2)

\begin{tabular}{|l|r|r|r|}
\hline \multicolumn{2}{|l|}{ Cost } & A1 & A2 \\
\hline Manufacturing & & & \\
TR04 find the last version of business object & 20 & & \\
TR05 increase productivity/ efficiency & 90 & & \\
TR16 notify changes & & & \\
Marketing & 80 & X & \\
TR14 notify changes & 20 & X & \\
TR15 find the last version of business object & 80 & X & X \\
Production Planning & 70 & X & X \\
TR01 anticipate design phase & 20 & X & X \\
TR02 evaluate retrofit policies & & & \\
TR03 find the last version of business object & 60 & & \\
Purchases & 40 & X & X \\
TR06 establish contractual constraints with supplier & 40 & & \\
TR07 give longer advance notice to supplier & & & \\
TR08 implement redundancy policy on supplier & & & \\
Quality Assurance & 25 & X & X \\
TR11 find version of the business object compliant with specification & & & \\
Sales under analysis & 20 & X & \\
TR12 find the last version of business object & 80 & X & X \\
TR13 notify changes & & & \\
Warehouse & 90 & X & X \\
\hline TR09 increase normalization & 525 & 485 \\
\hline TR10 know and apply concepts of effectiveness and versioning &
\end{tabular}

Once treatment operationalization is completed, analysts re-assess risks as illustrated in Section 5.2. In particular, they can analyze and evaluate different strategies (i.e., sets of treatments) to mitigate risks. Here, we have considered two strategies - A1 and A2 - as defined in Table $5^{1}$. Each strategy is associated with a cost (Total Cost in Table 5) which is defined as the sum of the cost of the treatments that compound it. In our example, A1 costs 525 and $\mathrm{A} 2$ costs 485 .

For each strategy, analysts determine if the residual risk is within the risk tolerance defined by actors. In other words, analysts evaluate the effectiveness of the treatments employed. Table 6 presents the residual risk calculated for different sets of treatments. As in Table 5, we use a "check", as in Table 4, if the residual risk is acceptable (e.g., $\mathrm{G}_{18}$ for Marketing), and a "cross" if not (e.g., $\mathrm{G}_{11}$ for Production Planning). Moreover, we use a down arrow to indicate that the residual risk is lower than the loss calculated before employing treatments, with the intended meaning that treatments have mitigated risks successfully; a right arrow to indicate that the residual risk is equal to the loss, with the intended meaning that treatments have no effect on risks; and a up arrow to indicate that the residual risk is higher than the initial loss, with the intended meaning that treatments have aggravated risks.

\footnotetext{
1 "X" refers to the treatment that is adopted to the system.
} 
Table 6: Residual Risk after Mitigation

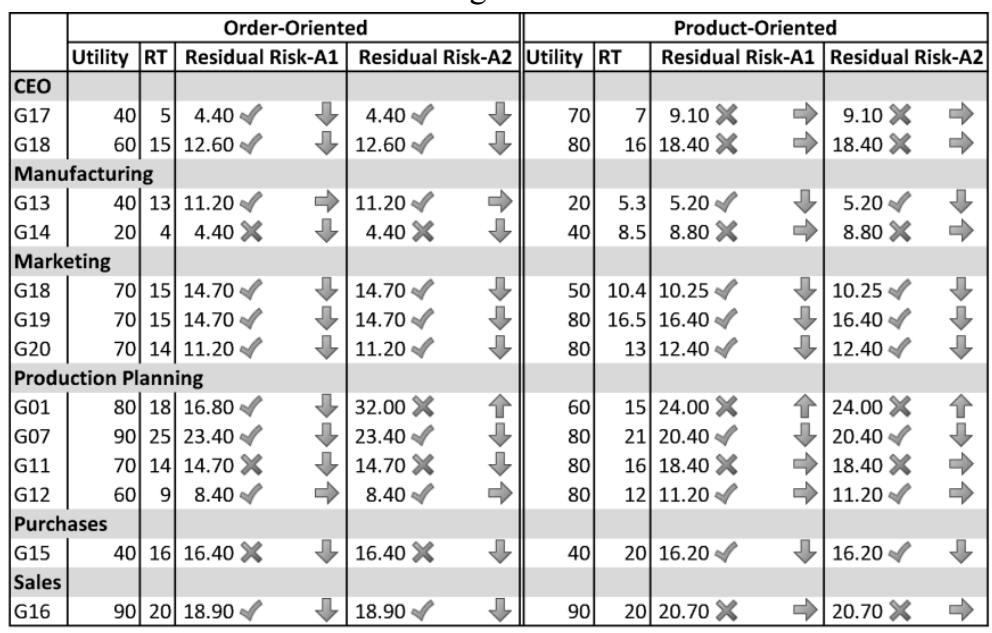

Example 12 - Table 4 shows that the loss of $\mathrm{G}_{16}$ exceeds the risk tolerance defined by the Sales division and the loss of $\mathrm{G}_{14}$ exceeds the risk tolerance defined by the Manufacturing division. In both cases, the application of A1 reduces the loss in orderoriented companies (Table 6). However, the residual risk of $\mathrm{G}_{14}$ is still too high for Manufacturing, whereas Sales reputes the adopted treatments sufficient to reduce risk to an acceptable level.

The adoption of different strategies may not necessary result in different level of residual risk, and the adoption of the same strategy may have a different effect over productoriented and order-oriented companies.

Example 13 - Though A1 costs more than A2, both strategies result in the same residual risk in product-oriented companies (Table 6).

Example 14 - In the PP division of order-oriented companies, the adoption of A1 reduces the loss of business goals $\mathrm{G}_{01}, \mathrm{G}_{07}$ and $\mathrm{G}_{11}$, and has negligible effects towards the loss of $\mathrm{G}_{12}$. In the same division of product-oriented companies, the adoption of $\mathrm{A} 1$ reduces the loss of $G_{07}$ only. It has negligible effects towards the loss of $G_{11}$ and $G_{12}$, and aggravates the loss of $\mathrm{G}_{01}$.

The framework allows analysts to perform cost-benefit analysis over alternative strategies by considering the cost of every strategy besides risks, and to adopt the most suitable solution. Analysts thus can negotiate a solution with the actors even if residual risk is higher than risk tolerance due to the cost of treatments. When actors cannot accept the residual risk, analysts need to identify additional treatments until the residual risk is acceptable.

\section{Discussion}

Starting from the study of the application domain and the performed analysis, we notice that SMEs are still human-centric. For instance, the knowledge, that would have to be 
corporate assets, concentrates in few and irreplaceable people. Moreover, the intrinsic complexity level of organizations is growing, though we are aware that there is no clearcut correspondence between the economic size of the company and its organizational complexity. This setting raises the scalability issues of the organization-size and, frequently, limits the dynamism needed for competing in a global market. Therefore, the tactical and strategic coordination choices among divisions are constrained by the people capability of synthesizing information that they have. As consequence, such information may not be complete and up-to-date. This makes it difficult to identify new opportunities, leading to the stagnation of organization growth.

The result of this work can be considered also in terms of the definition and application of Key Performance Indicators (KPIs) (Parmenter, 2007). They are metrics used to define and measure the progress toward organizational goals. For instance, considering the goal $\mathrm{G}_{17}$ reduce time-to-market in Table 1, a corresponding KPI is timeto-market. In order to instantiate our approach in a specific SME organization, we need to identify the KPIs of this organization. In this context, the aspects to consider essentially concern the definition of the organizational business processes meant as a collection of ordered tasks achieving a goal. Once these business processes have been defined, evaluation criteria of their performance must be identified and applied to compare them. In order to automatize the business processes based on KPIs, we need to define a requirements engineering methodology enabling to move from organizational requirements to system/software requirements (Abeti et. al., 2008). The presentation of this methodology is out of the scope of this paper.

The attempt to capture and analyze these challenges has allowed us to evaluate the expressiveness and usability of the GR framework and prove its applicability in industry. In particular, this study has pointed out a number of difficulties and benefits. The first difficulty concerns the selection of the most appropriate relations (i.e., alleviation, contribution, etc.) meant to capture and model the activities of an organization as well as their relationships with risks. Even though the semantics of such relations is well-defined, there are different ways to express the same scenarios of interest by using different combinations of relations as shown in the following example. 
Example 15 - To achieve the goal evaluate spare parts needed by customer $(\mathrm{G})$, the manufacturing department perform query to define diagnosis (T) that can be done either by query manufacturing $\left(\mathrm{T}_{01}\right)$, query warehouse $\left(\mathrm{T}_{02}\right)$, and [query external suppliers $\left(\mathrm{T}_{03}\right)$. This scenario can be depicted in two ways as presented in Figure 6.

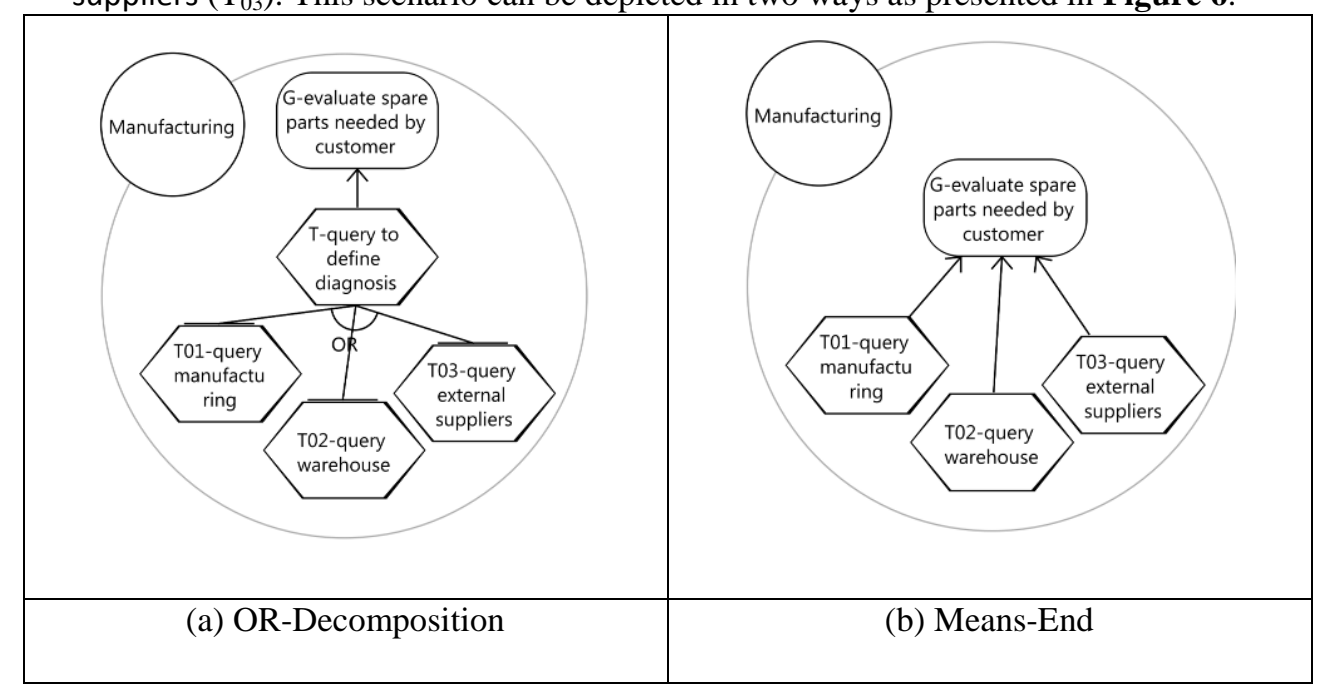

Figure 6: Alternative Ways of Modeling

Further investigation is necessary to evaluate modeling alternatives from the viewpoint of their equivalence and expressiveness.

The second difficulty is produced by the need of finding a rationale for the values assigned to the likelihood of events, the severity of events obstructing the goals, the cost of treatments, the utility associated to business goals, and the level of risk tolerance for each actor. Our rationale is based on the experience of the Think3's manager in interpreting the data from past projects. It would be interesting to investigate the validation of such values by considering a rigorous empirical analysis.

Last, but not least, the GR modeling framework suffers lack of scalability. Graphical models are getting harder to read following the increasing size of the models. This aspect is central since by nature the complexity of SMEs keeps growing proportionally to the growth of its products lifecycle.

As regards the benefits of the framework, we have experienced a remarkable easiness in capturing interdependencies among actors within an SME and analyze its consequences. This is a key factor to assist manufacturing organizations in optimizing their product development process by enhancing the integration, coordination, cooperation and interoperability among their divisions. For instance, it supports risk assessment within the whole organization setting by propagating risks across the interdependencies between actors.

This work was intended to find some critical aspects of manufacturing SMEs. The framework has resulted successful in the identification of some problematic requirements such as risky requirements and conflicting requirements. Part of the critical aspects identified in this work has been addressed by Think3 when developing software products for its clients. In addition, the framework enables a better capability of discussion about 
requirements among the involved stakeholders (i.e., managers, engineers, customers, etc.).

The complexity growth of SME product lifecycle makes the management of products and their related data more and more strategic in gaining the market. Consequently, many SMEs pay attention to research and development. To give an illustration, the design part of a product may involve the $15-20 \%$ of the company staff, especially for companies that deal with new custom-made markets. In these SMEs, the Research and Development division influences highly the time-to-market of a product. The design of products also determines the cost of the production process (e.g., $\pm 20 \%$ of costs can be affected because of the different production policies). In such situations, the GR framework can support analysts in analyzing alternative solutions to the design. Moreover, the framework can be applied in the early phase of solution development process, reducing the time-to-market for a new product.

The result of this work is currently driving the development of a Think3's Product Lifecycle Management (PLM) application with the aim of exploiting profitable organizational strategies pointed out by means of this approach. In particular, the acquired knowledge is used in the requirements engineering methodology (mentioned in Section 6) aiming to bridge the gap between the organizational requirements and the system/software requirements by preserving the benefits of the selected organizational strategies. As a result, we can exploit the tight relation between the tasks and treatments included in the selected strategies and the design and production processes that the PLM application enables to define.

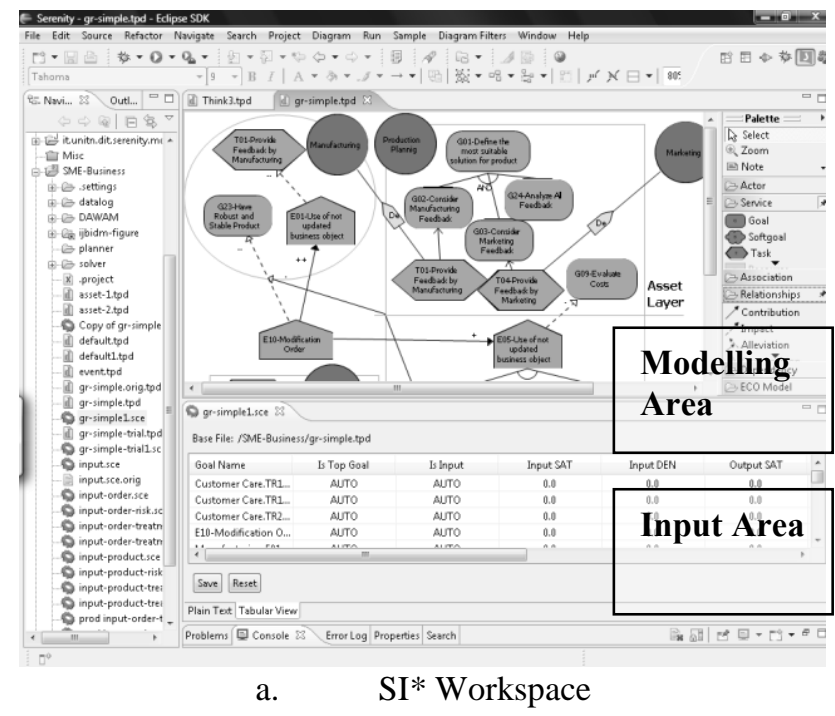




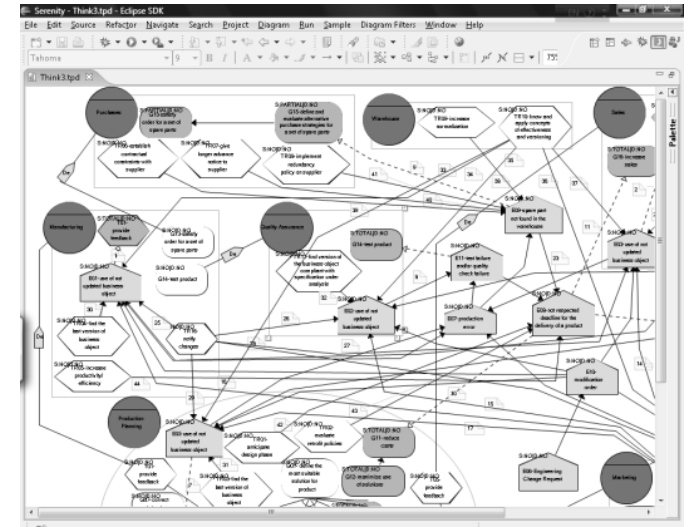

b. Assessement Result in a GR Model

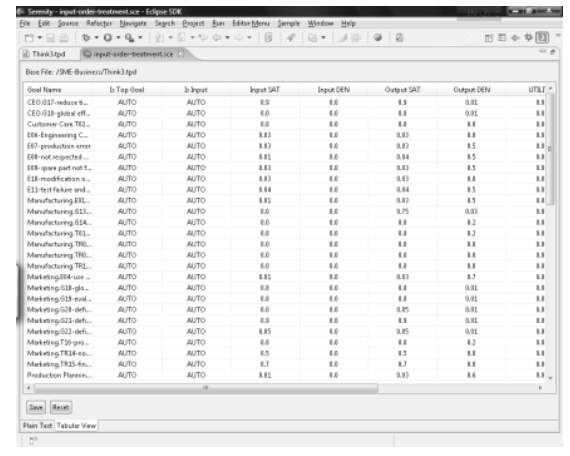

c. Detail of Assessement Result

Figure 7: $\mathrm{Si}^{*}$ Tool

Finally, even if it is not a commercial product, the framework is supported by a tool, the SI* Tool, ${ }^{1}$ that consists of a modeling environment and a reasoner. Screenshots of the tool are given in Figure 7. The tool is designed to assist analysts in executing all the phases of the risk management process described in this paper. Its graphical interface can be partitioned into two main areas (see Figure 7(a)): a Modeling Area where analysts can draw GR models, and an Input Area where analysts can specify the inputs of analysis. The tool also provide analysts with facilities to represent the output of the risk assessment process. In particular, the result of risk assessment is graphically represented as the degree of "greenness" of goals (see Figure 7(b)): a goal with green color is the one that suffer less risk. To obtain the detail result, analysts can see the output table as depicted in Figure 7(c). A complete study of the usability and expressiveness of the framework and the tool has been presented in Asnar et al. (2007).

In the point of view of performance, we have tested the performance of the risk assessment reasoner that is implemented using $\mathrm{Java}^{\mathrm{TM}}$ Runtime Environment (1.4.2) using "huge" GR models that are generated by multiplying and perturbing a GR model. The experiment indicates that the execution time grows exponentially with the size of the model (i.e., the number of nodes and the number of edges connecting nodes). For small models (i.e., below 50 nodes and 500 relations), the reasoner requires $15 \mathrm{~ms}$ to assess its risk. In larger models (i.e., 10.000 nodes with 100.000 relations), the reasoner needs $1 \mathrm{~s}$ to compute the risk level. Notice, the size of the GR model for this paper is 65 nodes with 200 relations (i.e., at this size, analysts can hardly understand the model if they view all nodes-relations of the model). From the experiments, we have deduced that the reasoner is more sensitive to the increase of relations more than the increase of nodes.

\section{Conclusions}

In this paper, we have presented the application of the Tropos Risk Management process for the analysis of risk in manufacturing SMEs. The process is intended to assess and treat risks within organizations. In this study, we have evaluated alternative business

${ }^{1}$ The tool is available at http://sesa.dit.unitn.it/sistar tool 
solutions, and chosen the most appropriate one according to the risk tolerance specified by stakeholders and its cost. However, the evaluation can be extended by including other criteria, such as time consumption. From this experience, we learned that "impact analysis" is the most critical step in the Tropos Risk Management process. It is because the result of this step drives both the outcome of risk assessment and treatment, and the business solution selection. Impact analysis is prone to subjective inputs that can lead to inappropriate decisions. Therefore, exploiting the past experience of SMEs (e.g., enterprise statistics, Log analysis etc.) is fundamental to assist analysts in performing this step correctly.

\section{Acknowledgments}

This work has been partially funded by the EU-SERENITY and EU-MASTER project, by the FIRB TOCAI.IT project, by the PRIN-MENSA project, and by the Canada's NSERC Hyperion project.

\section{References}

Abeti, L., Ciancarini, P., \& Moretti, R. (2008), 'Business process modeling for organizational knowledge management'. In Concurrency, Graphs and Models, volume 5065 of LNCS, (pp. 301-311). Springer.

Asnar, Y., Bonato, R., Giorgini, P., Massacci, F., Meduri, V., Riccucci, C., and Saidane, A. (2007), 'Secure and Dependable Patterns in Organizations: An Empirical Approach'. In Proc. of the 15th IEEE International Requirements Engineering Conference (RE'07), IEEE Press.

Asnar, Y. \& Giorgini, P. (2006) 'Modelling Risk and Identifying Countermeasures in Organizations', In Proc. of 1st Int. Workshop on Critical Inform. Infrastructures Sec. (CRITIS '06), volume 4347 of LNCS, (pp. 55-66). Springer.

Asnar, Y. \& Giorgini, P. (2007) 'Risk Analysis as part of the Requirements Engineering Process', Report DIT-07-014, DIT - University of Trento.

Asnar, Y., Moretti, R., Sebastianis, M., \& Zannone, N. (2008) 'Risk as Dependability Metrics for the Evaluation of Business Solutions: A Model-driven Approach', In Proc. Of $3^{\text {rd }}$ Int. Workshop on Dependability Aspects on Data Warehose \& Mining Application (DAWAM '08).

Bedford, T. \& Cooke, R. (2001), Probabilistic Risk Analysis: Foundations and Methods, Cambridge University Press.

Butler, S. \& Fischbeck, P. (2001), Multi-Attribute Risk Assessment, Technical Report CMU-CS-01169, Carnegie Mellon University.

Butler, S. A. (2002), 'Security Attribute Evaluation Method: a Cost-Benefit Approach', In Proc. of ICSE'02, (pp. 232-240)., New York, NY, USA. ACM Press.

Carr, M. J., Konda, S. L., Monarch, I., Ulrich, F. C., \& Walker, C. F. (1993), Taxonomy-Based Risk Identification, Technical Report CMU/SEI-93-TR-6, SEI-CMU.

den Braber, F., Dimitrakos, T., Gran, B. A., Lund, M. S., Stølen, K., \& Aagedal, J. Ø. (2003), 'The CORAS Methodology: Model-Based Risk Assessment using UML and UP', In UML and the Unified Process (pp. 332-357). Idea Group Publishing.

DoD (1980), Military Standard, Procedures for Performing a Failure Mode, Effects, and Critical Analysis, MIL-STD-1629A.

Feather, M. S. (2004), 'Towards a Unified Approach to the Representation of, and Reasoning with, Probabilistic Risk Information about Software and its System Interface', In Proc. of the 15th IEEE Int. Symp. on Soft. Soft. Reliability Eng., (pp. 391-402). IEEE CS Press. 
Feather, M. S., Cornford, S. L., Hicks, K. A., \& Johnson, K. R. (2005), 'Applications of Tool Support for Risk-Informed Requirements Reasoning', Computer Systems Science \& Engineering, 20(1).

Giorgini, P., Mylopoulos, J., \& Sebastiani, R. (2005), 'Goal-Oriented Requirements Analysis and Reasoning in the Tropos Methodology', Engineering Applications of Artificial Intelligence, 18(2), 159-171.

Goodman, P. (2004), Software Metrics: Best Practices for Successful IT Management, Rothstein Associates Inc., Publisher.

Holton, G. A. (2004), 'Defining Risk', Financial Analyst Journal, 60(6), 1925.

ISO/IEC Guide 73 (2002), Risk Management-Vocabulary-Guidelines for Use in Standards, ISO/IEC Guide 73.

Jaquith, A. (2007), Security Metrics: Replacing Fear, Uncertainty, and Doubt, Addison Wesley.

Kiyavitskaya, N., Moretti, R., Sebastianis, M., \& Zannone, N. (2007), Project Report on the Initial Analysis of (Early) Requirements of Domain, TOCAI Deliverable D2.1.

Landwehr, C. E., Bull, A. R., McDermott, J. P., \& Choi, W. S. (1994), 'Taxonomy of Computer Program Security Flaws', ACM Comp. Surveys, 26(3), 211-254.

Lee, S.-W., Gandhi, R., Muthurajan, D., Yavagal, D., \& Ahn, G.-J. (2006), 'Building Problem Domain Ontology from Security Requirements in Regulatory Documents', In Proc. of the 2006 Workshop on Soft. Eng. for Secure Sys. Building Trustworthy Applications, (pp. 43-50)., New York, NY, USA. ACM Press.

Lindsey, D., Cheney, P., Kasper, G. M., \& Ives, B. (1990, 'TELCOT: An application of information technology for competitive advantage in the cotton industry', MIS Quarterly, 14(4), 347-357.

Maizlitsh, B. \& Handler, R. (2005), IT Portfolio Management: Step by Step, Wiley \& Sons.

McFarlan, W. E. (1984), 'Information technology changes the way you compete', Harvard Business Review, 62(3), 98-103.

Parmenter, D. (2007), Key Performance Indicators. Wiley \& Sons.Pooley, R. \& Wilcox, P. (2000), 'Distributing decision making using Java simulation across the World Wide Web', Journal of the Operational Research Society, 51(4), 395-404.

Rico, D. F. (2004), ROI of Software Process Improvement: Metrics for Project Managers and Software Engineers, Ross Publishing.

Stamatelatos, M., Vesely, W., Dugan, J., Fragola, J., Minarick, J., \& Railsback, J. (2002), Fault Tree Handbook with Aerospace Applications, NASA. 